\title{
Antibacterial activity of Cinnamomum cassia L. essential oil in a carbapenem- and polymyxin-resistant Klebsiella aerogenes strain
}

\author{
Nathalie Gaebler Vasconcelos ${ }^{[1],[2], ~ K e ́ s i a ~ E s t h e r ~ S i l v a ~}{ }^{[1],}$ \\ Júlio Croda ${ }^{[3],[4]}$ and Simone Simionatto ${ }^{[1]}$
}

\author{
[1]. Universidade Federal da Grande Dourados, Laboratório de Pesquisa em Ciências da Saúde, Dourados, MS, Brasil. \\ [2]. Universidade Federal da Grande Dourados, Hospital Universitário de Dourados, Dourados, MS, Brasil. \\ [3]. Fundação Oswaldo Cruz, Campo Grande, MS, Brasil. [4]. Universidade Federal do Mato Grosso do Sul, Campo Grande, MS, Brasil.
}

\begin{abstract}
Introduction: Essential oils can serve as novel sources of antibiotics for multidrug-resistant bacteria. Methods: The multidrugresistance profile of a Klebsiella aerogenes strain was assessed by PCR and sequencing. The antibacterial activity of Cinnamomum cassia essential oil (CCeo) against $K$. aerogenes was assessed by broth microdilution and time-kill methods. Results: $K$. aerogenes showed high antibiotic resistance. The genes $b l a_{\mathrm{KPC}-2}, a m p C$, bla $a_{\mathrm{CTX}-\mathrm{M}-15}, b l a_{\mathrm{OXA}-1}$, and $b l a_{\mathrm{TEM}}$ were present. CCeo exhibited an inhibitory effect with a minimum inhibitory concentration of $17.57 \mu \mathrm{g} / \mathrm{mL}$. Conclusions: The antibacterial activity of CCeo makes it a potential candidate for treating carbapenem- and polymyxin-resistant $K$. aerogenes strains.
\end{abstract}

Keywords: Drug resistance. Cinnamon oil. Carbapenem-resistant Enterobacterales. Gram-negative bacteria. Klebsiella aerogenes. Polymyxin resistance.

The emergence of multidrug-resistant bacteria is an important public health issue. Klebsiella aerogenes, previously known as Enterobacter aerogenes, has become an important opportunistic pathogen that often causes hospital-acquired infections such as pneumonia, urinary tract infections, bacteremia, and surgical site infections ${ }^{1}$. Worldwide, the number of infections caused by $K$. aerogenes strains with acquired resistance to most classes of commercially available antimicrobial agents, including carbapenems and polymyxins, is rising ${ }^{2}$. Carbapenems are often used to treat serious infections caused by $K$. aerogenes, especially those that produce $\mathrm{AmpC}$ cephalosporinases or extended-spectrum $\beta$-lactamases $(\mathrm{ESBL})^{1}$, but carbapenem resistance can also arise. This scenario has led us to reconsider polymyxins as valuable therapeutic options. However, in addition to carbapenem resistance, various genera have demonstrated the ability to acquire polymyxin resistance ${ }^{3}$.

\footnotetext{
Corresponding author: Dra. Simone Simionatto.

e-mail: simonesimionatto@ufgd.edu.br

(D)https://orcid.org/0000-0003-2367-0915.

Received 31 January 2020

Accepted 13 May 2020
}

Carbapenemase-producing pathogens that are also resistant to polymyxins are a matter of concern, as these characteristics drastically reduce therapeutic options ${ }^{4}$. Carbapenem- and polymyxin-resistant Enterobacterales are considered critical by the World Health Organization, which has thus listed research into and development of new antibiotics as a high priority.

Polymyxin-resistant $K$. aerogenes strains have been identified and are being increasingly reported ${ }^{5}$. This highlights the urgency to find novel treatment options for carbapenem- and polymyxinresistant bacterial infections. Molecular analysis of such strains may help improve our understanding of the antimicrobial resistance mechanisms and allow for the development of new and effective antibiotics.

With an increasing number of antibiotics rendered ineffective, the study of medicinal plants as potential sources of novel antibiotics, especially for the treatment of multidrug-resistant infections, has increased ${ }^{5}$. Plant-derived essential oils contain potent natural agents, known as secondary metabolites, that have been used for hundreds of years against pathogens ${ }^{6}$. An important characteristic in essential oils is their hydrophobicity, which allows them to interact with the lipids in the bacterial cell membrane and 
disturb the bacterial structure, thereby making it more permeable and leading to the leakage of ions and various molecules from the bacterial cells. Although the extravasation of cellular components can be tolerated without loss of viability, further loss of cellular content or the loss of critical molecules and ions can lead to cell death ${ }^{4}$. Cinnamomum cassia L. (Lauraceae) essential oil (CCeo), popularly known as cinnamon, contains a wide variety of secondary metabolites and has been shown to exhibit antimicrobial effects? In this study, the antimicrobial activity of $\mathrm{CCeo}$ in the inhibition of a carbapenem- and polymyxin-resistant $K$. aerogenes strain was evaluated.

CCeo is a yellow to yellow brown clear oily liquid, extracted from leaves, bark, and branches by steam distillation, with a density of $1.053 \mathrm{~g} / \mathrm{cm}^{3}$. CCeo originated in China, and this compound was acquired commercially from Ferquima (Vargem Grande Paulista, SP, Brazil) (CAS number: 84961-46-6). According to the chromatographic technical report provided by the supplier, cinnamaldehyde was the most prevalent compound (87.6\%), followed by $\alpha$-humulene (3.1\%), $\gamma$-elemene (2.5\%), borneol (1.5\%), cinnamic acid $(0.7 \%)$, benzaldehyde $(0.5 \%)$, and eugenol $(0.4 \%)$, and other minor components (3.7\%) (Figure 1).

A clinical isolate of a carbapenem- and polymyxin-resistant $K$. aerogenes was identified in September 2015 in a microbiological vigilance culture (nasal swab) at a tertiary Brazilian hospital. The isolate was identified using BD Phoenix 100 (Becton Dickinson, Franklin Lakes, NJ) and the antimicrobial susceptibility was determined by broth microdilution and by using BD Phoenix 100. Preliminary screening for the presence of carbapenemase was performed using the modified Hodge test ${ }^{8}$. The study was conducted with the approval of the Research Ethics Committee of the Universidade Federal da Grande Dourados (Process no. 877.292/2014).

Polymerase chain reaction (PCR) and sequencing were performed to determine the presence of the carbapenems-resistance genes $b l a_{\text {CTX-M-1-like, }}, b l a_{\text {CTX-M-2-like }}, b l a_{\text {CTX-M-8-like }}, b l a_{\text {CTX-M-14-like }}, b l a_{\text {GES-like' }}$ $b l a_{\mathrm{GIM}-\mathrm{like}}, b l a_{\mathrm{IMP}-10}, b l a_{\mathrm{IMP}-\mathrm{like}}, b l a_{\mathrm{KPC}-2}, b l a_{\mathrm{NDM}-\mathrm{like}}, b l a_{\mathrm{OXA}-23}, b l a_{\mathrm{OXA}-48-}$ like, $b l a_{\text {SHV-like, }}, b l a_{\text {SIM-like, }}, b l a_{\text {SME-like, }}, b l a_{\text {SPM-like, }}, b l a_{\text {TEM-like, }}$, and $b l a_{\text {VIM-like }}^{2}$, and the polymyxin B-resistant gene $m c r-1^{5}$.

Genomic DNA was extracted from a fresh culture. The concentration and purity of the DNA were determined with a Qubit 2.0 fluorometer using the dsDNA BR Assay Kit (Life Technologies, Carlsbad, CA). A sequencing library was prepared using the Nextera library kit (Illumina, San Diego, CA). The DNA sample was sequenced using the Illumina MiSeq Platform (Illumina), as described by Dung et al. ${ }^{9}$ Readings were mapped to a reference sequence, and species identification was confirmed using the taxonomic classification system of Kraken ${ }^{10}$. The reading set was assembled using SPAdes version 3.6.1 and annotated using the software tool Prokka ${ }^{11}$. The reading set was also screened for known alleles using a reading mapping approach with SRST2, the Short Read Sequence Typing for Bacterial Pathogens. For the acquired resistance genes, the Antibiotic Resistance Gene-ANNOTation (ARG-ANNOT) database was used ${ }^{12}$.

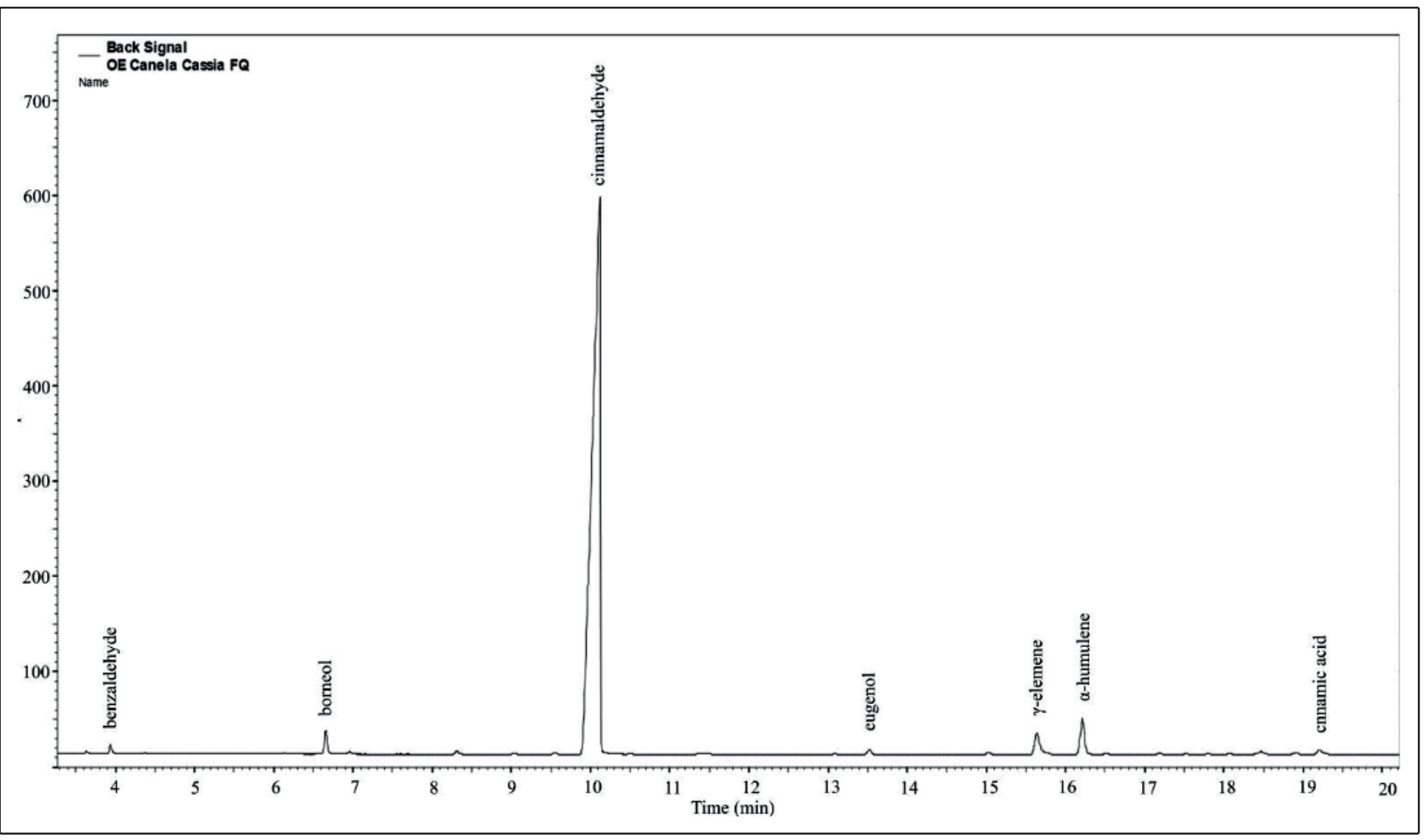

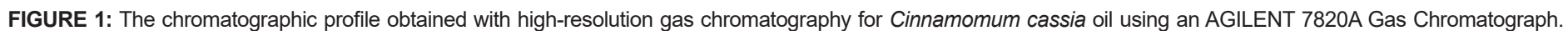

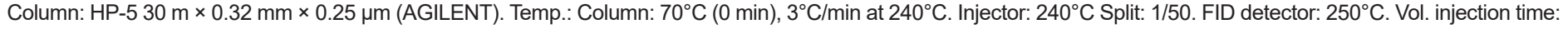

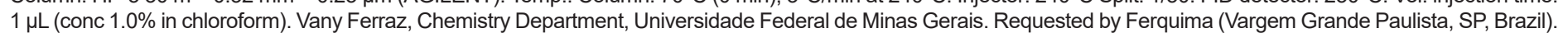


The minimum inhibitory concentration (MIC) of CCeo was determined by broth microdilution and by using a resazurin colorimetric assay ${ }^{13}$. The tested concentration range of CCeo was $72,000 \mu \mathrm{g} / \mathrm{mL}-2.197 \mu \mathrm{g} / \mathrm{mL}$. The time-kill method was performed using the broth macrodilution technique ${ }^{14}$. The concentration of $\mathrm{CCeo}$ and polymyxin B tested represented the MIC value. Timekill studies were performed with approximately $1.5 \times 10^{6} \mathrm{CFU} / \mathrm{mL}$. A positive control of tigecycline was used at twice the MIC value, since the studied strain had an intermediate sensitivity to this antibiotic and there was no sensitivity to other pharmacological classes. A negative control of brain heart infusion broth was used for the bacterial strain, and saline was used as sterility control.

The $K$. aerogenes strain has an ESBL-, carbapenem- and polymyxin-resistant profile (Table 1). The genomic data assembled using ResFinder identified 17 resistance genes. The metallo- $\beta$ lactamase resistance genes identified were $a m p C, b l a_{\mathrm{CTX}-\mathrm{M}-15}, b l a_{\mathrm{KPC}-2}$, $b l a_{\text {OXA-1 }}$, and $b l a_{\text {TEM }}$. Other resistance genes observed in this strain, including aac3, aac6, aphA6, catB4, dfrA8, dfrA14, qnrB1, strA, $s t r B$, sul2, tet $A$, and tet $R$, conferred resistance to aminoglycosides, chloramphenicol, trimethoprim, fluoroquinolones, streptomycin, sulfamethoxazole, and tetracycline. The genes $b l a_{\text {CTX-M-1-like }}, b l a_{\text {CTX- }}$ M-2-like, $b l a_{\text {CTX-M-8-like }}, b l a_{\text {CTX-M-14-like }}, b l a_{\text {GES-like, }}, b l a_{\text {GIM-like }}, b l a_{\text {IMP-10 }}, b l a_{\text {IMP- }}$ ${ }_{\text {like, }}, b l a_{\text {NDM-like, }}, b l a_{\text {OXA-23 }}, b l a_{\text {OXA-24, }}, b l a_{\text {OXA-48, }} b l a_{\text {OXA-48-like }}, b l a_{\text {OXA-51, }}$

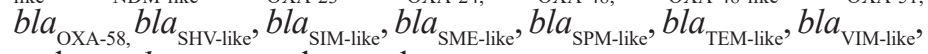
and $m c r-1$ were not detected.

Antimicrobial resistance to carbapenems and polymyxin B was confirmed. Molecular analysis was used to identify the $b l a_{\mathrm{KPC}-2}$ gene, elucidating the mechanism of carbapenem resistance. Sequencing also allowed us to identify other metallo- $\beta$-lactamase genes, such as ampC, bla $a_{\mathrm{CTX}-\mathrm{M}-15}, b l a_{\mathrm{OXA}-1}$, and $b l a_{\mathrm{TEM}}$, which provide $K$. aerogenes with a wide range of resistance mechanisms, possibly capable of transferring related genes, leading to an increase resistance to antibiotics, thus making it very difficult to treat infections caused by this bacterial strain. Resistance to polymyxin B due to the presence of $\mathrm{mcr}-1$ gene was not considered. However, sequencing results showed alterations in the $p h o P$ gene, indicating a potential mechanism of mutational colistin resistance in this strain.

CCeo exhibited an inhibitory effect against the carbapenem- and polymyxin-resistant $K$. aerogenes strain even at a low concentration $(0.0019 \% \mathrm{v} / \mathrm{v})$ and had a MIC value of $17.57 \mu \mathrm{g} / \mathrm{mL}$. The survival curve of the strain showed a decrease in viable cell counts over time (Figure 2). CCeo treatment decreased cell counts by approximately $5 \log _{10} \mathrm{CFU} / \mathrm{mL}$. The results showed total inhibition of the carbapenem- and polymyxin-resistant $K$. aerogenes, with cell counts reduced to zero after $24 \mathrm{~h}$ of CCeo treatment. Furthermore, polymyxin B showed no activity for up to $24 \mathrm{~h}$. Tigecycline was used at twice the MIC value as a positive control and was found to inhibit the strain successfully within $24 \mathrm{~h}$.

CCeo demonstrated promising antibacterial properties against the carbapenem- and polymyxin-resistant $K$. aerogenes strain, a bacterial strain resistant to almost all classes of clinical antibiotics, with a low MIC and a consistent action. To the best of our knowledge, this is the first report on the antibacterial activity of CCeo against multidrug-resistant $K$. aerogenes. In addition, to determine MIC values, an improved time-kill method was used,
TABLE 1: Antimicrobial susceptibility patterns (MICs in $\mu \mathrm{g} / \mathrm{mL}$ ).

\begin{tabular}{lc}
\hline Antibiotics & MIC (interpretation) \\
\hline Amikacin & $32(\mathrm{I})$ \\
Aztreonam & $>16(\mathrm{R})$ \\
Ceftazidime & $>16(\mathrm{R})$ \\
Cefepime & $>16(\mathrm{R})$ \\
Ceftriaxone & $>32(\mathrm{R})$ \\
Ciprofloxacin & $>2(\mathrm{R})$ \\
Ertapenem & $>4(\mathrm{R})$ \\
Gentamicin & $\leq 2(\mathrm{R})$ \\
Imipenem & $8(\mathrm{R})$ \\
Meropenem & $8(\mathrm{R})$ \\
Piperacillin/Tazobactam & $>64(\mathrm{R})$ \\
Polymyxin B & $>4(\mathrm{R})$ \\
Tigecycline & $2(\mathrm{I})$ \\
Sulfamethoxazole/Trimethoprim & $>4(\mathrm{R})$ \\
\hline
\end{tabular}

(I): intermediate; (R): resistance; MIC: minimum inhibitory concentration.
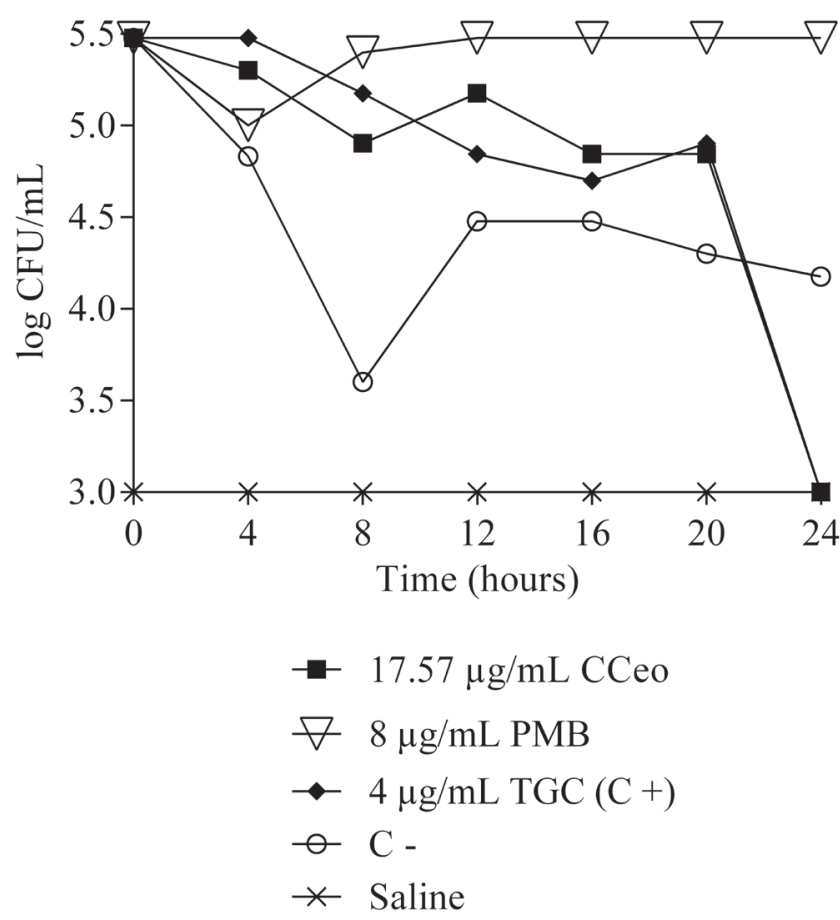

FIGURE 2: Time-kill curves with CCeo against a carbapenem and polymyxinresistant $K$. aerogenes strain.

CCeo: Cinnamomum cassia L. essential oil; MIC: minimum inhibitory concentration; PMB: polymyxin B; TGC: tigecycline; C+: positive control (tigecycline at twice the MIC value); C-: negative control $(K$. aerogenes and brain heart infusion broth).

which allowed us to verify the speed of the inhibition activity of $\mathrm{CCeo}$ on the studied bacteria, to enhance our understanding of the underlying mechanism of action of CCeo.

Our results suggest that the CCeo antibacterial action mechanism is complex. Several pathways may be involved in 
the inhibitory effects of this oil against bacterial cells, making it difficult to elucidate them all. Multiple experiments are necessary in order to evaluate all possible mechanisms and provide an insight into the molecular actions of CCeo. The antimicrobial activity of essential oils may involve single or multiple targets. Therefore, the mechanisms of action cannot be attributed to a unique site, but rather to a cascade of reactions involving the entire bacterial cell ${ }^{6}$.

Regarding CCeo toxicity, the major components of C. cassia are considered to be non-toxic and safe, with no acute or chronic toxicity, no mutagenicity or genotoxicity, and no carcinogenicity detected in mammalian studies ${ }^{15}$, making CCeo even more suitable for use as raw material in drug development.

Effective antimicrobial activity was observed at low concentrations, indicating that $\mathrm{CCeo}$ is a favorable candidate for in vivo studies in animal models to demonstrate its clinical applicability for the future development of antimicrobial treatments. Our findings fall in line with the critical need for novel sources of antibiotics to address an increasing incidence of drug-resistant pathogens, especially multidrug-resistant bacteria. However, this study had some limitations. Studies on other multidrug-resistant bacteria and in vivo studies are necessary to better understand the mechanisms of action of CCeo.

Our data reveal that the CCeo had an antibacterial effect, making it an interesting candidate for the development of therapeutic options against carbapenem- and polymyxin-resistant $K$. aerogenes strains. Further studies on CCeo activity in animals infected with multidrug-resistant bacteria are essential in order to further improve our understanding of its actions and establish its efficacy.

\section{ACKNOWLEDGMENTS}

The authors are grateful for the financial support and infrastructure provided by the Universidade Federal da Grande Dourados (UFGD), which made this study possible, and Hospital Universitário da Universidade Federal da Grande Dourados (HU-UFGD) for providing the microbial strain used in the study. K.E.S. received a scholarship from Coordenação de Aperfeiçoamento de Pessoal de Nível Superior (CAPES). This study was conducted with the approval of the Research Ethics Committee of the Universidade Federal da Grande Dourados (no. 877292/2014).

\section{FINANCIAL SUPPORT}

This work was partially supported by the National Council for Scientific and Technological Development (CNPq), Fundação de Apoio ao Desenvolvimento do Ensino, Ciência e Tecnologia do Estado de Mato Grosso do Sul (FUNDECT grants 092/2015 and 041/2017), Coordenação de Aperfeiçoamento de Pessoal de Nível Superior (CAPES, grant 001), and Universidade Federal da Grande Dourados (UFGD). K.E.S. received a scholarship from CAPES.

\section{AUTHORS' CONTRIBUTION}

All authors contributed to the study conception and design. Material preparation, data collection and analysis were performed by NGV and KES. JC and SS supervised the research. The first draft of the manuscript was written by NGV, and all authors commented on previous versions of the manuscript. All authors read and approved the final manuscript.

\section{CONFLICT OF INTEREST}

The authors declare no conflicts of interest.

\section{REFERENCES}

1. Shen X, Liu L, Yu J, Cao X, Zhan Q, Guo Y, et al. Coexistence of bla ${ }_{\text {NDM-1 }}$ and $r m t C$ on a Transferrable Plasmid of a Novel ST192 Klebsiella aerogenes Clinical Isolate. Infect Drug Resist. 2019;12:3883-91. doi: 10.2147/IDR.S228130.

2. Silva KE, Cayô R, Carvalhaes CG, Sachi FPC, Rodrigues-Costa F, Ramos da Silva AC, et al. Coproduction of KPC-2 and IMP-10 in Carbapenem-Resistant Serratia marcescens Isolates from an Outbreak in a Brazilian Teaching Hospital. J Clin Microbiol. 2015;53(7):2324-8. doi: 10.1128/JCM.00727-15.

3. da Silva KE, Baker S, Croda J, Nguyen TNT, Boinett CJ, Barbosa LS, et al. Risk factors for polymyxin-resistant carbapenemase-producing Enterobacteriaceae in critically patients: An epidemiological and clinical study. Int J Antimicrob Agents. 2020;55(3):105882. doi: 10.1016/j.ijantimicag.2020.105882.

4. Nazzaro F, Fratianni F, De Martino L, Coppola R, De Feo V. Effect of Essential Oils on Pathogenic Bacteria. Pharmaceuticals. 2013;6(12):145174. doi: 10.3390/ph6121451.

5. Liu YY, Wang Y, Walsh TR, Yi LX, Zhang R, Spencer J, et al. Emergence of plasmid-mediated colistin resistance mechanism MCR-1 in animals and human beings in China: a microbiological and molecular biological study. Lancet Infect Dis 2016;16(2):161-8. doi: 10.1016/S14733099(15)00424-7.

6. Vasconcelos NG, Croda J, Simionatto S. Antibacterial mechanisms of cinnamon and its constituents: A review. Microb Pathog 2018;120:198203. doi: 10.1016/j.micpath.2018.04.036.

7. Yap PS, Krishnan T, Chan KG, Lim SH. Antibacterial Mode of Action of Cinnamomum verum Bark Essential Oil, Alone and in Combination with Piperacillin, Against a Multi-Drug-Resistant Escherichia coli Strain. J Microbiol Biotechnol 2015;25(8):1299-306. doi: 10.4014/jmb.1407.07054.

8. Clinical Laboratory Standards Institute. Performance Standards for Antimicrobial Susceptibility Testing; Twenty-Five Informational Supplement. CLSI Document M100-S25; 2015.

9. Dung TTN, Duy PT, Sessions OM, Sangumathi UK, Phat VV, Tam PTT, et al. A universal genome sequencing method for rotavirus A from human fecal samples which identifies segment reassortment and multigenotype mixed infection. BMC Genomics 2017;18(1):324. doi: 10.1186/ s12864-017-3714-6.

10. Wood DE, Salzberg SL. Kraken: ultrafast metagenomic sequence classification using exact alignments. Genome Biol 2014;15(3):R46. doi: 10.1186/gb-2014-15-3-r46.

11. Seemann T. Prokka: rapid prokaryotic genome annotation. Bioinformatics 2014;30(14):2068-9. doi: 10.1093/bioinformatics/btu153.

12. Gupta SK, Padmanabhan BR, Diene SM, Lopez-Rojas R, Kempf M, Landraud L, et al. ARG-ANNOT, a new bioinformatic tool to discover antibiotic resistance genes in bacterial genomes. Antimicrob Agents Chemother 2014;58(1):212-20. doi: 10.1128/AAC.01310-13.

13. Cavalcanti YW, Almeida LFD, Padilha WWN. Antifungal Activity of Three Essential Oils on Candida Strains. Rev Odontol Bras Central 2011;20(52):68-73. doi: 10.1155/2017/7158756.

14. Vasconcelos NG, Croda J, Silva KE, Motta MLL, Maciel WG, Limiere LC, et al. Origanum vulgare L. essential oil inhibits the growth of carbapenem-resistant gram-negative bacteria. Rev Soc Bras Med Trop 2019;52:e20180502. doi: 10.1590/0037-8682-0502-2018.

15. Bickers D, Calow P, Greim H, Hanifin JM, Rogers AE, Saurat JH, et al. A toxologic and dermatologic assessment of cinnamyl alcohol, cinnamaldehyde and cinnamic acid when used as fragrance ingredients. The RIFM expert panel. Food Chem Toxicol 2005;43:799-836. doi: 10.1016/j.fct.2004.09.013. 